\title{
Urachal mucinous neoplasms-to be or not to be: Two cases and a review of current diagnostic criteria (WHO 2016)
}

\author{
Juan Daniel Prieto Cuadra*1,2, Lidia Pérez ${ }^{1}$, Isabel García ${ }^{1}$, Martina Álvarez ${ }^{3}$, Bernardo Herrera ${ }^{4}$, Isabel Hierro ${ }^{1}$, Luis \\ Vicioso $^{1}$ \\ ${ }^{1}$ Unidad de Gestión Clínica Provincial de Anatomía Patológica, Hospital Universitario Virgen de la Victoria, Málaga, Spain \\ ${ }^{2}$ LABCO Pathology, Malaga, Spain \\ ${ }^{3}$ Departamento de Anatomía Patológica. Facultad de Medicina. Universidad de Málaga, Málaga, Spain \\ ${ }^{4}$ Unidad de Gestión Clínica de Urología. Hospital Universitario Virgen de la Victoria, Málaga, Spain
}

Received: April 11, 2018

DOI: $10.5430 /$ crcp.v5n1p12
Accepted: May 16, 2018

Online Published: May 28, 2018

\begin{abstract}
The urachus is an embryological remnant that extends from the bladder to the umbilicus. Urachal neoplasms represent less than $1 \%$ of malignant bladder tumors. The new 2016 edition of disease classifications from the WHO has updated the classification of epithelial neoplasms of urachal origin, distinguishing between glandular and non-glandular neoplasms and between cystic and non-cystic adenocarcinomas. In this article, we present two cases with similar clinical presentations but differing histology. We address their morphological, immunohistochemical, and molecular characterization; review the classification and diagnostic criteria of glandular urachal neoplasms included in the most recent literature with the aim of clarifying their differential diagnosis as well as their prognostic implication; and, ultimately, better understand these infrequent biological entities.
\end{abstract}

Key Words: Urachus, Mucinous neoplasm, Urachal cyst, Adenocarcinoma, Immunohistochemistry, Molecular

\section{INTRODUCTION}

The urachus is an extraperitoneal fibromuscular band that extends from the bladder dome to the umbilicus. ${ }^{[1]}$ It is an embryological remnant connecting the urinary bladder to the allantois that obliterates following birth. ${ }^{[2]}$ This urachal remnant consists of an epithelial canal surrounded by connective tissue and smooth muscle layer. ${ }^{[3]}$ This epithelium tends to be urothelial, but the vast majority of urachal carcinomas are glandular (normally adenocarcinoma). ${ }^{[4]}$ This suggests that they could originate from intestinal metaplasia and posterior malignant transformation. ${ }^{[5]}$
Urachal carcinoma tends to grow from the bladder dome in the bladder-urachus junction. They are a rare neoplastic entity that represents less than $1 \%$ of all malignant urinary bladder tumors and between 10\%-20\% of bladder adenocarcinomas. ${ }^{[6,7]}$

It was described for the first time in 1863 by Hue and Jacquin. ${ }^{[8]}$ Since then, the terminology used for the subtypes had been varied until the World Health Organization (WHO) codified the different variants in the blue book published in 2004. ${ }^{[9]}$ Recently, in the WHO's fourth edition of disease classifications, published in 2016, the classifications Spain. 
of epithelial neoplasms of urachal origin have been updated in order to make their diagnosis more reproducible.

Clinical manifestations tend to derive from tumor invasion of neighboring structures. ${ }^{[4]}$ The majority of the tumors grow in the submucosa or muscularis propria layer, without invading the bladder mucosa in the first stages. ${ }^{[10]}$

Due to the fact that urachal adenocarcinoma progresses with slightly different symptoms and an ill-fated prognosis, it tends to be considered more as a clinical entity that is independent from the rest of bladder tumors. ${ }^{[11,12]}$

\section{Case PResentation}

\subsection{Case 1}

A 55-year-old woman who was a smoker and presented with irritative urinary symptoms, lumbar, abdominal pain, and gross hematuria. CT scan showed a lesion located in the urachal region. The cystoscopy showed a lesion in the dome that had a neoplastic appearance. Therefore, it was decided to perform a Transurethral resection biopsy (TURB). It showed a neoplasm composed of glands covered by columnar, mucinsecreting epithelial cells with marked pleomorphism and atypical mitosis alternating with pools of extracellular mucin. This suggests that it was a moderately-differentiated intestinal adenocarcinoma with mucinous areas (see Figure 1A-B).
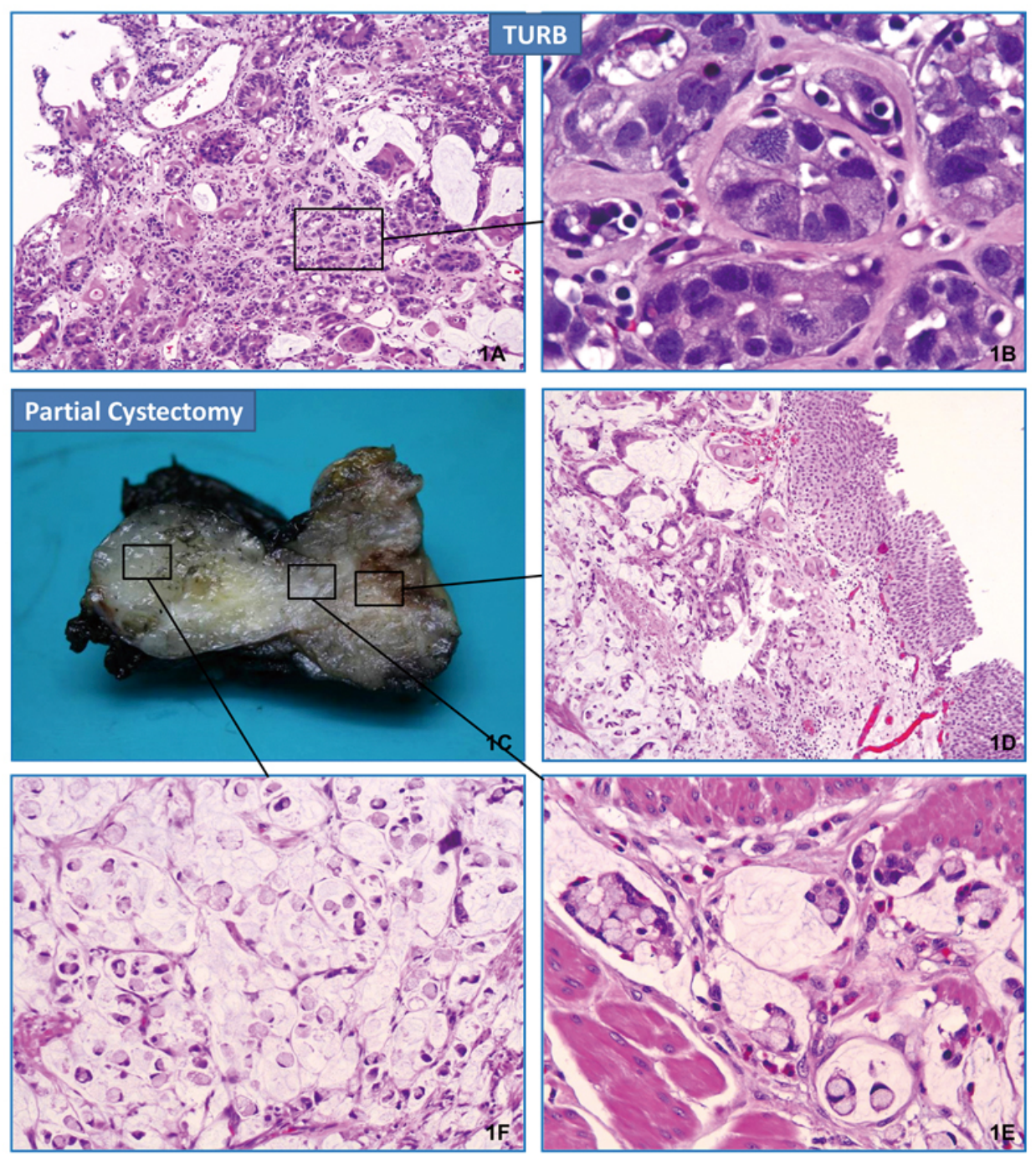

Figure 1. Pathological findings from Case 1. 1A-B: TURB fragments with mucinous adenocarcinoma and presence of structural and cytological atypia $(\mathrm{H} \& \mathrm{E}, 40 \times$ and $63 \times)$. 1C: Macroscopic image of a partial cystectomy showing the bladder dome (mucosa and muscular wall) connected to an oval-shaped pseudocystic structure with mucinous content. It appears to infiltrate the bladder wall. 1D: Bladder mucosa and submucosa infiltrated by mucinous adenocarcinoma (H\&E, 40×). 1E: Infiltration of the muscularis propria layer by mucinous adenocarcinoma with mucin pools and signet ring cells (H\&E, $400 \times$ ). 1F: Abundant mucinous pools with the presence of signet ring-type neoplastic cells $(\mathrm{H} \& \mathrm{E}, 200 \times)$. 
A partial cystectomy was performed. Grossly, this piece included an adjacent urachal structure that measured $5 \mathrm{~cm}$. No macroscopic lesions were observed in the bladder mucosa. A whitish-yellow, shiny, translucent, well-delimited solid nodular tumor with a maximum diameter of $2.5 \mathrm{~cm}$ was observed to be fixed to the external border of the muscle wall of the bladder (see Figure 1C). On the histopathological study, this nodular tumor was composed of mucin pools and signet ring cells (see Figure $1 \mathrm{~F}$ ). It transmurally infiltrated the bladder wall (see Figure 1E) and the submucosa (the supra-adjacent bladder epithelium was intact) in the form of mucinous adenocarcinoma (see Figure 1D) with presence of signet ring cells (isolated or in clusters). No glandular cystitis, intestinal metaplasia, or foci of dysplasia/carcinoma in situ were observed in the peritumoral bladder mucosa. Neither a healthy residual urachal structure nor involvement of the surgical margins were identified.
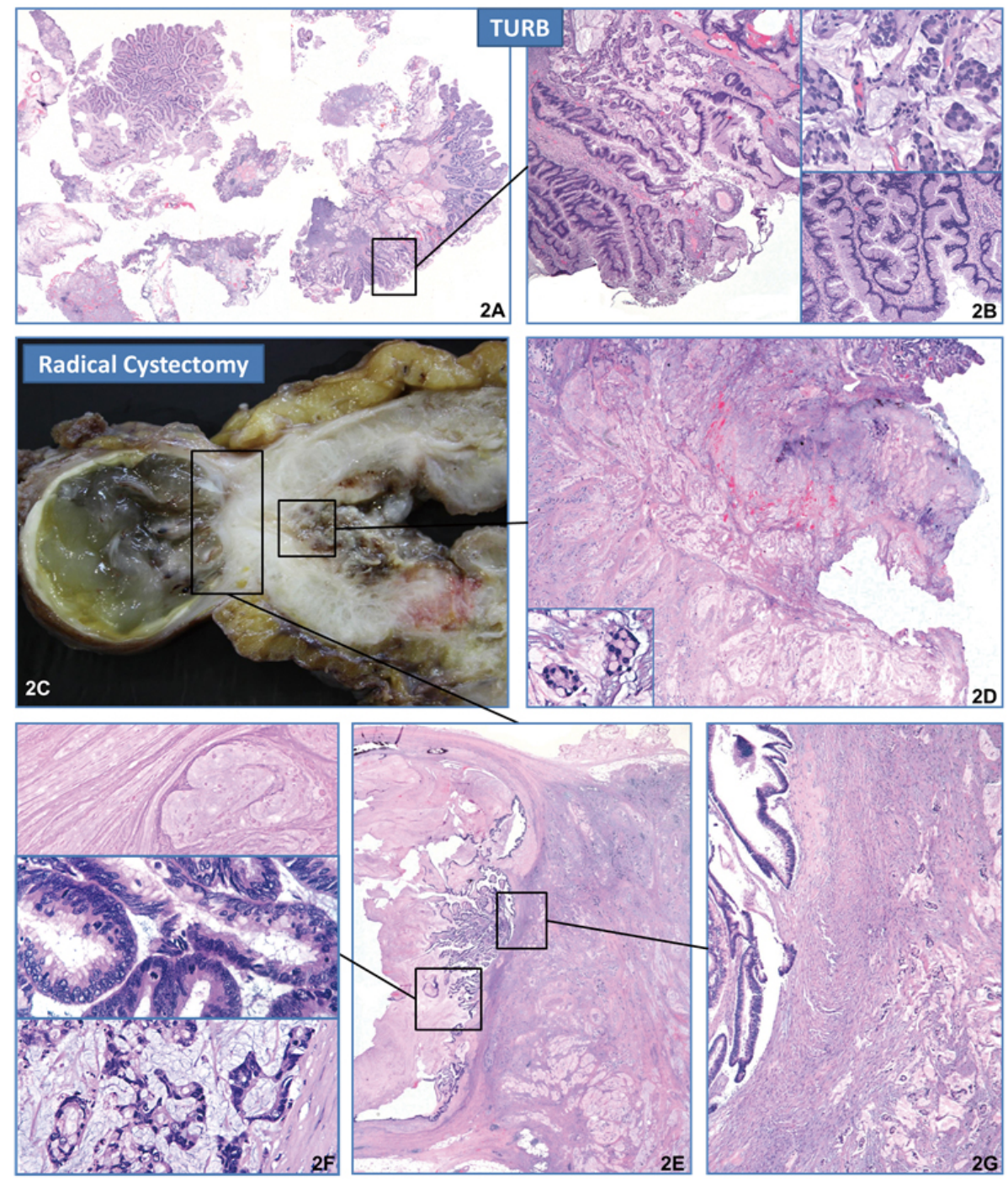

Figure 2. Pathological findings from Case 2. 2A-B: TURB fragments with mucinous adenocarcinoma and presence of structural and cytological atypia with adjacent areas of intestinal metaplasia $(H \& E, 20 \times, 40 \times, 200 \times, 400 \times)$. 2C:

Macroscopic image of a radical cystectomy. It has a cystic structure with a whitish wall and mucinous content adhered to the anterior wall of the bladder. It does not appear to infiltrate the bladder wall. 2D: Mucosa and bladder wall occupied by infiltrating mucinous material with the presence of clusters of signet ring cells ( $\mathrm{H} \& \mathrm{E}, 40 \times, 400 \times$ ). 2E: Area of transition between the bladder wall and cyst implantation base (H\&E, 20×). 2F: Cyst composed of abundant mucinous pools and fibrous wall partially covered with non-infiltrating intestinal metaplastic epithelium: It shows pseudostratification and mild atypia and focal presence of epithelial plaques with high-grade atypia, mitosis, and signet ring cells. (H\&E, 200×, 400×). 2G: Fibrous band free of neoplasms between the epithelium of the mucinous cystic tumor and the infiltrating neoplastic foci of the primary mucinous adenocarcinoma of the bladder 
On the immunohistochemical study, the neoplastic cells showed immunoexpression for cytokeratins (CK7: weak and focal; CK20; diffuse), CDX-2, and beta-catenin in cytoplasmic and cellular membrane patterns, thus confirming the urothelial origin of the neoplasia. Likewise, they showed conserved expression of the four DNA Mismatch Repairs Proteins (pMMR): MLH-1, MSH-2, MSH-6, and PMS-2 (see Figure 3).
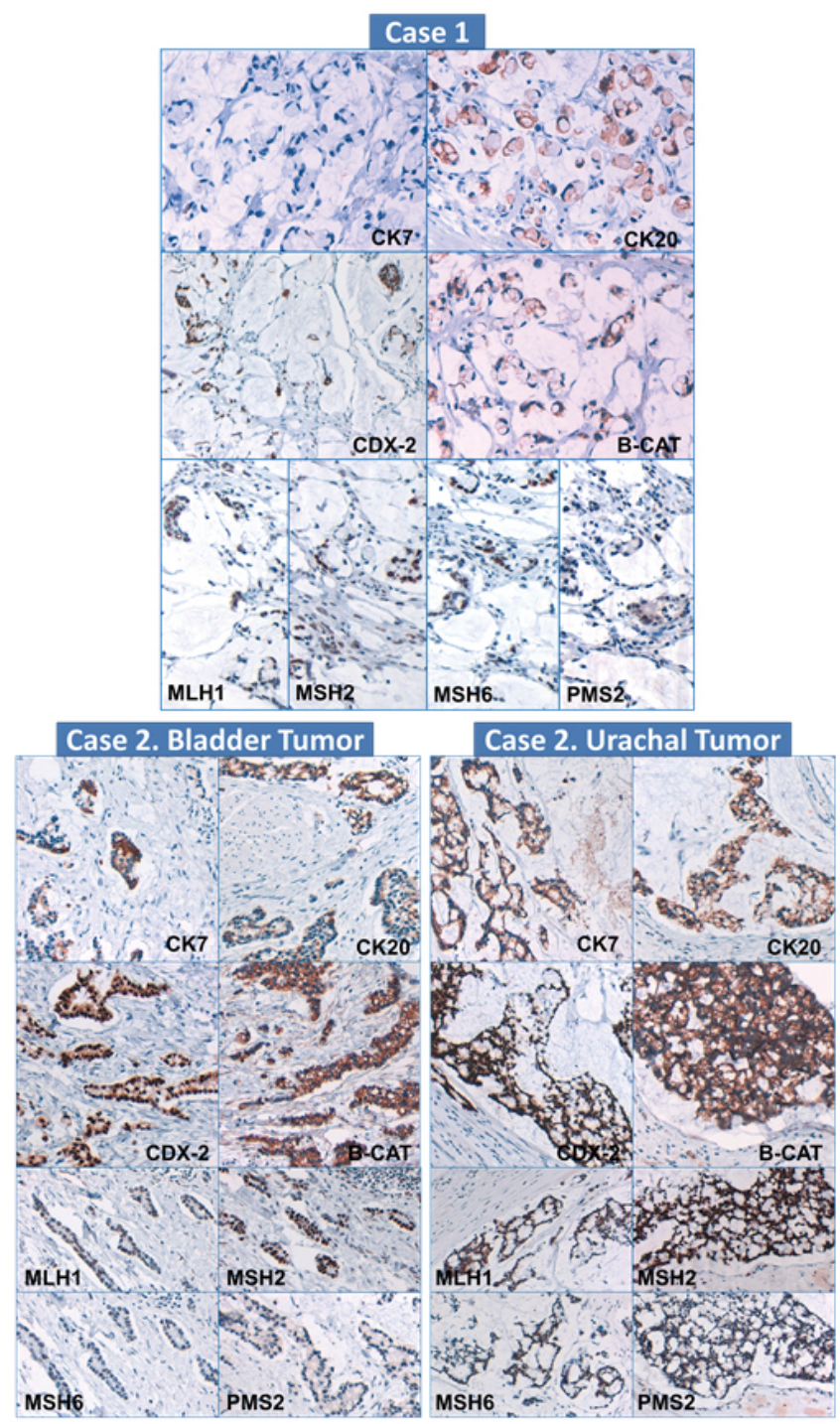

Figure 3. Immunohistochemical findings from both cases. Cytokeratin 7, Cytokeratin 20, CDX-2, $\beta$-catenin, and mismatch repair (MMR) proteins (MLH1, MSH2, MSH6, and PMS2)

The molecular study was carried out in the Laboratorio de Biología Molecular del Cáncer [Molecular Biology of Cancer Laboratory] (CIMES, University of Málaga), where IDylla technology based on quantitative PCR was used to analyze the mutational state of KRAS, NRAS, and BRAF. No mutations in those genes were detected.

Afterwards, a massive sequencing study was performed using the Trusight Tumor 15 assay from Illumina. Multiple genes were analyzed (AKT1, BRAF, EGFR, ERBB2, FOXL2, GNA11, GNAQ, KIT, KRAS, MET, NRAS, PDGFRA, PIK3CA, RET, and P53). Only a P53 mutation was detected (p.Cys141Arg; c.421T>C, variant allele frequency: 0.268).

The decision was made not to give chemotherapy and a monitoring approach was adopted. Currently, the patient is in follow-up and is disease-free four years after surgery.

\subsection{Case 2}

A smoker and type II diabetes mellitus 60-year-old woman patient presented urinary incontinence, and hematuria. Radiologically (URO-CT), a lesion on the bladder and urachal wall was observed. On the cystoscopy, a large bladder neoformation with mucoid and fibrin areas. A TURB was performed (see Figures 2A, 2B). The tissue showed a neoplasm composed of superficial glandular formations with intestinal metaplasia-type and atypia (see Figure 2B, lower right) and transition zones to predominantly-mucinous primary adenocarcinoma that, in depth, formed infiltrating pools of mucin with clusters of signet right cells in their interior (see Figure 2B, upper right).

In the piece from the radical cystectomy, an excrescent, irregular lesion on the bladder mucosa that measured $2.2 \mathrm{~cm}$ and was of a clear, brownish-grey color was observed. It had an irregular surface and appeared to infiltrate the muscle wall without affecting the peribladder fat. A cystic lesion measuring $3.3 \mathrm{~cm}$ with yellowish mucinous content and whitish walls was also noted. It was located in the anterior peribladder fat area, at $2 \mathrm{~cm}$ from the bladder tumor lesion (see Figure 2C).

Microscopically, it was observed that the superficial borders of the neoplasm were composed of glandular adenocarcinoma with areas of intestinal metaplasia, whereas the main central component was abundant extracellular mucin. In depth, the neoplasm displayed a pattern that was mainly mucinous adenocarcinoma with signet ring cells, isolated and in clusters/plaques, that floated in mucin pools and infiltrated the bladder wall (see Figure 2D), remaining adjacent but with no signs of continuity with the extrabladder mucinous cystic structure (see Figure 2E, 2G).

This urachal cystic structure was composed of a thick fibrous wall with dystrophic calcification. There were no signs of epithelial entrapment or mucin extravasation in its interior. It was partially covered by a non-infiltrating, mucinous, pseudostratified columnar epithelium with mitosis and cytological 
atypia that varied from mild to severe (see Figure 2F middle) with areas of cribriform patterns with signet ring cells (see Figure $2 \mathrm{~F}$ lower) as well as abundant unilocular mucinous content with no signs of floating neoplastic cells (see Figure 2F upper).

On the immunohistochemical study, both tumor components (the primary bladder tumor and the mucinous cystic structure) showed a similar immunohistochemical profile. They had immunoexpression for cytokeratins (CK7, CK20), CDX-2, and beta-catenin of a cytoplasmic and cellular membrane pattern, thus confirming the urothelial origin of both neoplasms. Likewise, both components showed conserved expression of the four DNA mismatch repair proteins (see Figure 3).

From a molecular point of view, both tumor components were analyzed as independent samples using the same technology (Idylla and Trusight Tumor 15). Just as in the above case, only a P53 mutation was detected. However, it was a different variant (p.Arg248Gln; c.743G $>$ A, variant allele frequency: 0.762) and it was in both samples.

The Oncology team decided to opt for monitoring without any adjuvant therapy for this patient. Currently, the patient is in follow-up and is disease-free two years after surgery.

\section{Discussion}

The diagnosis criteria for differentiating between adenocarcinomas of urachal origin and primary bladder adenocarcinomas were first described by Wheeler and Hill in 1954. ${ }^{[13]}$ These criteria were later modified by Sheldon et al., ${ }^{[14]}$ who proposed the classification system that is most recognized today, but these criteria are too restrictive. ${ }^{[15]}$

Recently, other criteria have been proposed by Gopalan et al. ${ }^{[16]}$ and Dhillon et al. ${ }^{[17]}$ These includes some that are already known, such as a) location of the tumor in the anterior wall or fundus; b) absence of another known primary tumor; c) epicenter in the bladder wall and; d) absence of cystitis glandularis in the bladder surface. They also include two new criteria: e) absence of urothelial neoplasms with glandular differentiation in the rest of the bladder and f) absence of atypical intestinal metaplasia.

We observe that these criteria are met in our first case. However, the bladder neoplasm in the second case is excluded due to the presence of a mucinous adenocarcinoma-type primary urothelial neoplasia with adjacent areas of atypical intestinal metaplasia.

The WHO classification (2016) distinguishes between glandular and non-glandular neoplasms and likewise differentiates between cystic and non-cystic adenocarcinomas. ${ }^{[5,18]}$ The majority of urachal epithelial neoplasms are non-cystic urachal adenocarcinomas. They are subdivided into the following subtypes: enteric, mucinous or colloid, mixed, of signet ring cells, or unspecified. ${ }^{[9]}$

The mucinous or colloid subtype must have predominance of mucin pools with scattered or clustered malignant epithelial cells floating in their interior. In the mixed subtype, there should not be a predominant pattern whereas in the "unspecified" subtype, the lesion should not show a classifiable histology. ${ }^{[3]}$ The neoplastic cells may have intracytoplasmic mucin and scattered or aggregated signet ring cells ("clusters") may be observed floating in the mucin pool. ${ }^{[19]}$

Our first case corresponds to a mucinous subtype, which is characterized by pools of extracellular mucin with signet ring cells that are scattered or in nests floating in its interior. Mucinous cystic tumors are classified in a similary manner to mucinous cystic neoplasms of the ovary. The classification proposed by the WHO includes several entities, including the Mucinous Cystic Tumor of Low Malignant Potential with Intraepithelial Carcinoma (MCTLMP-IC). ${ }^{[3,5]}$

Due to the morphological diversity, a wide range of possibilities must be taken into account in the differential diagnosis. For example, enteric non-cystic urachal adenocarcinoma is morphologically indistinguishable from colorectal adenocarcinoma. The mucinous subtype can be confused with appendiceal mucinous adenocarcinoma, pancreatobiliary adenocarcinoma, or adenocarcinoma of ovarian origin. Likewise, the signet ring cell subtype imitates similar tumors of gastric, colorectal, or ovarian origin. ${ }^{[5]}$ The use of markers such as CDX2, CK7, CK20, and $\beta$-catenin and other such as CEA, EMA, racemaces, estrogen and progesterone receptors, and E-cadherin ${ }^{[8]}$ has been recommended to differentiate urachal adenocarcinomas from those of other origins. ${ }^{[20,21]}$

For all of this, when faced with bladder biopsies or transurethral resections that display any subtype and are of uncertain primary origin, it is recommended to use the term "infiltrating adenocarcinoma of the bladder" and, in a comment, explain the correlation with the clinical data and imaging so that clinical specialists can determine the origin with the greatest possible certainty. With respect to our cases, both showed very similar immunohistochemical profiles, despite the fact that they come from both the bladder mucosa and the urachal structure (cystic and non-cystic).

Molecular studies have demonstrated the presence of KRAS mutations in 39\% of adenocarcinomas of the urachus studied. Of these, $100 \%$ were of the mucinous subtype. ${ }^{[22]}$ In another study, microsatellite inestability (MSI) was observed. In one case, MSH2-MSH6 loss was shown and in others, PMS2 loss was shown. ${ }^{[5,23]}$ However, wider cohort studies are re- 
quired in order to validate these findings and define more specific molecular profiles. In our cases, we have not observed molecular abnormalities in KRAS or in other common targets except for TP53.

In regards to the disease staging, Amin et al. ${ }^{[3]}$ suggest not staging according to the system proposed by Sheldon et al. ${ }^{[14]}$ Amin suggests that the most important thing would perhaps be to only categorize them in two large groups: disease restricted to the surgical piece (negative margins) versus disease that has disseminated intraperitoneally. Surgical treatment is variable, though a partial cystectomy with a complete urachal resection and umbilectomy is the most common. ${ }^{[3]}$ The role of neoadjuvant or adjuvant chemotherapy has not yet been defined. ${ }^{[5]}$

Invasive non-cystic urachal carcinoma has a poor prognosis. The most important independent predictive factor for survival is surgical margin status. In comparison with bladder urothelial carcinomas with similar staging, urachal adenocarcinoma has longer mean cancer-specific survival, develops at younger ages, has low-grade lesions, and has a greater probability of distant metastases. ${ }^{[7]}$ Despite this, our two cases had a therapeutic focus that only differed in regards to surgical resection extension, but both have the same favorable prognosis.

\section{Conclusions}

In this article we present two cases of urachal mucinous neoplasms that display different histopathological characteristics. The first case is an example of non-cystic urachal mucinous adenocarcinoma with signet ring cells.
The second case does not meet the criteria to be considered a single primary urachal tumor as it had two independent neoplasms: a primary mucinous adenocarcinoma in the bladder and a urachal mucinous cystic neoplasm of low malignant potential with foci of with intraepithelial carcinoma. Following extensive inclusion, we have confirmed that both are adjacent but independent neoplasms and they show very different morphology and degrees of atypia. Given the presence of intestinal metaplasia in both, our impression is that they come from the same biological origin, have progressed in a parallel manner, and, in the end, produced similar mucinous neoplasms. This hypothesis is also supported by the presence of a similar immunohistochemical expression and molecular mutation profiles in both components.

It has yet to be clarified if the presence of mutations in different TP53 codons is related to the genesis or progression of urachal mucinous neoplasms. Likewise, it remains to be investigated if the presence of intestinal metaplasia as a precursor lesion could be equally related to the aforementioned mutational profile. For all of this, extensive, multi-centric studies are required to attempt to improve the characterization of these biological entities and their prognostic implications prognostic implications.

\section{ACKNOWLEDGeMents}

Claire Alexandra Conrad provided language help and translation.

\section{CONFLICTS OF INTEREST DISCLOSURE}

No conflicts of interest.

\section{REFERENCES}

[1] Hammong GYL, Davis JE. The urachus, its anatomy and associated fasciae. Anat Rec. 1941; 80: 271-87. https://doi.org/10.100 2/ar. 1090800302

[2] Schubert GE, Pavkovic MB, Bethke-Bedurftig BA. Tubular urachal remnants in adult bladders. J Urol. 1982; 127: 40-42. https: //doi.org/10.1016/S0022-5347(17) 53595-8

[3] Amin MB, Smith SC, Eble JN. Glandular neoplasm of the urachus: a report of 55 cases emphasizing mucinous cystic tumors with the proposed classification. Am J Surg Pathol. 2014; 38: 1033-1045. PMid:25025366. https://doi.org/10.1097/PAS. 0000000000 000250

[4] Paner GP, BarKan GA, Mehta V. Urachal carcinomas of the nonglandular type: salient features and considerations in pathologic diagnosis. Am J Surg Pathol. 2012; 36: 432-442. PMid:22301493. https://doi.org/10.1097/PAS.0b013e31823fe49c

[5] Paner GP, Lopez-Beltrán A, Sirohi D. Updates in the Pathologic diagnosis and classification of epithelial neoplasm of urachal ori- gin. Adv Anat Pathol. 2016; 23: 71-83. PMid:26849813. https: //doi.org/10.1097/PAP.0000000000000110

[6] Bruins J, VisserO, Ploeg M. The clinical epidemiology of urachal carcinoma: results of a large, population based study. J Urol. 2012; 188: 1102-1107. PMid:22901574. https://doi.org/10.1016/j. juro.2012.06.020

[7] Wright JL, Porter MP, Li CL. Differences in survival among patients with urachal and nonurachal adenocarcinomas of the bladder. Cancer. 2006; 107: 721-728. PMid:16826584. https://doi.org/10.100 2/cncr. 22059

[8] Hue and Jacquin. Cancer coloide de lómbilic et de la paroi abdominale anterieure ayant envahi la vessie. Union med. 1983; 6: 418.

[9] Ayala A, Tamboli P. Urachal carcinoma. In International Agency for Research on Cancer. Pathology and genetics of tumours of the urinary system and male genital organs. Lyon: IARC Press; 2004. $131 \mathrm{p}$.

[10] Zong L, Chen P. Surgical and chemotherapeutic experience regarding a urachal carcinoma with repeated relapse: case report and literature review. World Journal of surgical oncology. 2013; 11: 170- 
8. PMid:23914849. https://doi.org/10.1186/1477-7819-1 $1-170$

[11] Ojea A, Núñez A, Domínguez F. Adenocarcinoma mucinoso de uraco. Actas Urol Esp. 2003; 27(2): 142-146. https : //doi .org/10.101 6/S0210-4806 (03) 72892-7

[12] Murphy WM, Grignon DJ, Perlman EJ. Tumors of the kidney, bladder, and related urinary structures. AFIP atlas of tumor pathology. Series 4. Washington DC: ARP Press; 2008.

[13] Sheldon CA, Clayman RV, González R. Malignant urachal lesions. J Urol. 1984; 131: 1-8. https : //doi .org/10.1016/S0022-534 7 (17) $50167-6$

[14] Wheeler JD, Hill WT. Adenocarcinoma involving the urinary bladder. Cancer. 1954; 7: 119-135. https://doi.org/10.1002/1097-0 142(195401) 7:1<119: :AID-CNCR2820070113>3.0.CO;2-8

[15] Johnson DE, Hodge GB, Abdul-Karim FW, et al. Urachal carcinoma. Urology. 1985; 26: 218-221. https://doi.org/10.1016/0090 -4295 (85) $90112-8$

[16] Gopalan A, Sharp DS, Fine SW. Urachal carcinoma: a clinicopathologic analysis of 24 cases with outcome correlation. An J Surg Pathol. 2009; 33 (5): 659-68. PMid:19252435. https://doi.org/10.1 097/PAS . Ob013e31819aa4ae
[17] Dhillon J, Lian Y, Kamat AM. Urachal carcinoma: a pathologic and clinical study of 46 cases. Hum Pathol. 2015; 46: 1808-1814. PMid:26364859. https ://doi.org/10.1016/j.humpath. 2015 .07 .021

[18] Lopez-Beltran A, Paner G, Tsuzuki T. Urachal carcinoma. In International Agency for Research on Cancer. Pathology and genetics of tumours of the urinary system and male genital organs. Lyon: IARC Press; 2016. 113 p.

[19] Chen CY, Li MH, Tsai WM. Signet ring cell adenocarcinoma of the urachus: a case report and review of 28 cases. JTUA. 2006; 17 : 103-8.

[20] Singh I, Prasad R. Primary urachal mucinous adenocarcinoma of the urinary bladder. Journal of Clinical and Diagnostic Research. 2013; 7(5): 911-13. PMid:23814741.

[21] Dadhania V, Czerniak B, Gui CC. Adenocarcinoma of the urinary bladder. Am J Clin Exp Urol. 2015; 3(2): 51-63. PMid:26309895.

[22] Hutchinson LPCG, Tomaszewicz K, Mehta K. KRAS is mutated in a subset of mucinous type urachal adenocarcinomas. Mod Pathol. 2012; 25: 214A.

[23] Sirantrapun SJ, Ward M, Woo J. High-stage urachal adenocarcinoma can be associated with microsatellite instability and KRAS mutations. Hum Pathol. 2014; 45: 327-330. PMid:24355196. https: //doi.org/10.1016/j.humpath.2013.09.008 